\title{
Common Roadblocks for Biomaterials Metrologists
}

\author{
Michael Wininger ${ }^{1,2,3}$ \\ 1 Prosthetics \& Orthotics Program, University of Hartford; West Hartford, CT 06117, USA; \\ wininger@hartford.edu; Tel.: +1-860-768-5787 \\ 2 Department of Biostatistics, Yale School of Public Health; New Haven, CT 06520-8034, USA \\ 3 Cooperative Studies Program, Department of Veterans Affairs, West Haven, CT 06516, USA \\ Academic Editor: Pankaj Vadgama \\ Received: 14 April 2016; Accepted: 16 May 2016; Published: 18 May 2016
}

In this issue, Naylor et al. [1] report on the surface topography of prosthetic phalanges, important research that is increasingly vital to researchers and clinicians alike. While their approach is within the conventions of the field, and therefore cannot be impugned, this publication evidences common roadblocks for biomaterials metrologists.

Naylor et al.'s paper (J. Funct. Biomater. 2016, 7, 9, doi:10.3390/jfb7020009) looks at a single specimen per phalanx; this is an inherent limitation, and speaks to the utter difficulty in establishing adequate sample size for estimating error and asserting homogeneity and repeatability. Naylor et al.'s paper also poses two suggestions about the putative impact of polishing and lubrication on these implants; where these conjectures are unaccompanied by supporting justification, e.g., demonstration of scale compatibility between a polishing tool and lubricant at the surface, shows that there remains much to be learned about the physicochemical stresses at the biomaterial interface.

Lastly, we note the use of surface roughness measures root-mean-square (RMS), skewness and kurtosis. These parameters measure the variability of feature height across the sample surface relative to the arithmetic mean. These methods were not invented by Naylor et al.; rather, these measures have been promulgated over the past 35 years by institutions of the highest repute in governmental and industrial standards-making, including the NIST [2], NASA [3], the National Physical Laboratory-UK [4], and the British Standards Institute (ISO-7206-2) [5].

This population-average approach is limited by the lack of spatial information. Roughness is an inherently "neighborly: concept, where "smooth" reflects a small height difference between adjacent loci, and "rough" reflects a large difference in height between adjacencies. To account for spatial relationships, topographic measures should account for spatial variables. As an example, we consider the conventional measures as posed in Naylor et al., e.g., skewness:

$$
\delta \propto \int_{X} \int_{Y}\left(\frac{z(x, y)-\mu}{\sigma}\right)^{3} \mathrm{~d} x \mathrm{~d} y
$$

(Skewness)

where $z$ is the surface height measured at $x \in X$ and $y \in Y$, and note its spatial insensitivity: the integrand considers only the global parameters $\mu$ and $\sigma$ : the global mean and standard deviation of feature heights across the sample. Now consider a modest re-formulation that incorporates height differences between adjacent loci:

$$
\rho \propto \int_{X} \int_{Y}\left(\left(\frac{\mathrm{d}^{3}}{\mathrm{~d} x^{3}} z(x, y)\right)^{2}+\left(\frac{\mathrm{d}^{3}}{\mathrm{~d} y^{3}} z(x, y)\right)^{2}\right) \mathrm{d} x \mathrm{~d} y
$$


where $\frac{\mathrm{d}^{3}}{\mathrm{~d} x^{3}} z(x, y)$ and $\frac{\mathrm{d}^{3}}{\mathrm{~d} y^{3}} z(x, y)$ are the instantaneous rate of change of the "acceleration" of feature height $z$ with respect to spatial variables $x$ and $y$. This is the spatial analogue to the jerk common to researches in robotics, biomechanics, and motor control [6,7].

We show the performance of skewness $\delta$ and roughness $\rho$ on two simulated surfaces with clearly distinguishable roughness profiles (Figure 1). Consider a completely smooth surface (upper panel) and the same surface with all loci randomly reorganized (lower panel). The population average (and therefore skeweness) is identical in both images, but the spatially sensitive roughness is starkly different.

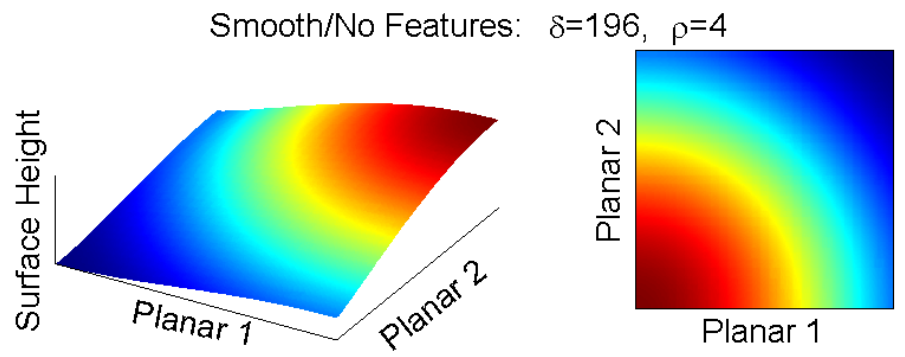

(A)

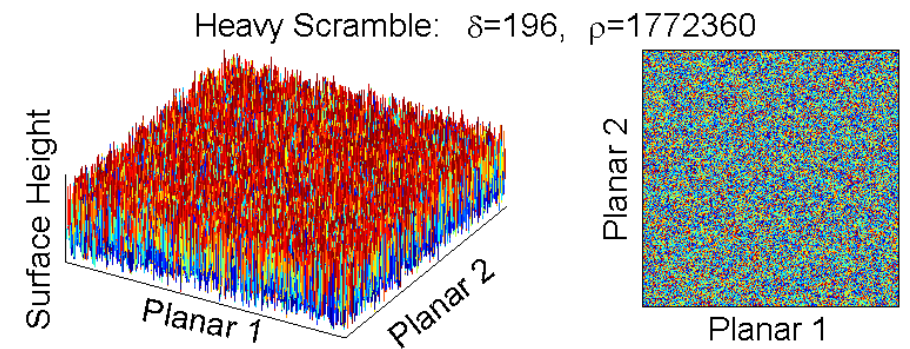

(B)

Figure 1. Skewness $(\delta)$ and roughness $(\rho)$ for two simulated surfaces. Smooth surface $(\mathbf{A})$ and smooth surface randomly re-configured ("scrambled") into a rough surface (B). Both surfaces contain the same set of pixels; consequently, $\delta$ remains the same in both samples (sample mean $\mu$ and standard deviation $\sigma$ do not change). However, the height differences between adjacent loci are captured via use of the derivative in $\rho$; as a result, the calculated roughness matches the visual profile.

It is only fair to view Naylor et al.'s approach in the context of established practices. Additionally, for that matter, prudence must be used in applying jerk to a dataset, as whether and how to normalize jerk remains an open line of inquiry [8-10]; even the most theoretically sound approach requires validation and revision through implementation. Nevertheless, research enterprise in metrology must adhere to the same empirical best practices expected elsewhere in science: using adequate sample size and proper statistical design, a well-posed analytical approach, and scrutable interpretations. It should be strongly encouraged that studies based on a single specimen be withheld until additional samples can be collected and analyzed, and that investigators refrain from unsupported speculation. In particular as it concerns material science, we must immediately revoke the use of distribution-based measures in characterizing spatially sensitive phenomena (i.e., RMS, skewness and kurtosis as a measure of surface roughness), and revisit conclusions drawn from extant studies where these flawed practices were adopted. Indubitably, we should advise the standard-makers to revise their guidance documents so as to eliminate the error at its source.

Conflicts of Interest: The author declares no conflict of interest. 


\section{References}

1. Naylor, A.; Talwalkar, S.C.; Trail, I.A.; Joyce, T.J. Evaluating the Surface Topography of Pyrolytic Carbon Finger Prostheses through Measurement of Various Roughness Parameters. J. Funct. Biomater. 2016, 7, 9. [CrossRef] [PubMed]

2. Vorbuger, T.V. Methods of Characterizing Surface Topography. Tutorials in Optics; Moore, D.T., Ed.; Optical Society of America: Washington, DC, USA, 1992; pp. 138-142. Available online: http://www.nist.gov/ calibrations/upload/osa-92.pdf (accessed on 16 May 2016).

3. Li, C.J.; Devries, W.R.; Ludema, K.C. Classification of Journal Surfaces Using Surface Topography Parameters and Software Methods to Compensate for Stylus Geometry; Technical Report Accession Number 83NS4857. National Aeronautics and Space Administration (NASA): Washington, DC, USA, 1983; pp. 9-12. Available online: http:/ /ntrs.nasa.gov/archive/nasa/casi.ntrs.nasa.gov/19830016586.pdf (accessed on 16 May 2016).

4. Leach, R.; Brown, J.; Jiang, X.; Blunt, R.; Conroy, M. Guide to the Measurement of Smooth Surface Topography Using Coherence Scanning Interferometry; Measurement Good Practice Guide No. 108; National Physical Laboratory: Middlesex, UK, 2008; pp. 16-19. Available online: http://publications.npl.co.uk/npl_web/pdf/ mgpg108.pdf (accessed on 16 May 2016).

5. BS EN ISO 7206-2 Implants for Surgery_Partial and Total Hip Joint Prostheses. Part 2: Articulating Surfaces Made of Metallic, Ceramic and Plastics Materials; British Standards Institute: London, UK, 2011.

6. Hogan, N. An Organizing Principle for a Class of Voluntary Movements. J. Neurosci. 1984, 4, $2745-2754$. [PubMed]

7. Flash, T.; Hogan, N. The Coordination of Arm Movements: An Experimentally Confirmed Mathematical Model. J. Neurosci. 1985, 5, 1688-1703. [PubMed]

8. Wininger, M.; Kim, N.-H.; Craelius, W. Spatial Resolution of Spontaneous Accelerations in Reaching Tasks. J. Biomech. 2009, 42, 29-34. [CrossRef] [PubMed]

9. Hogan, N.; Sternad, D. Sensitivity of Smoothness Measures to Movement Duration, Amplitude, and Arrests. J. Mot. Behav. 2009, 41, 529-534. [CrossRef] [PubMed]

10. Wininger, M.; Kim, N.-H.; Craelius, W. Reformulation in the Phase Plane Enhances Smoothness Rater Accuracy in Stroke. J. Mot. Behav. 2012, 44, 149-159. [CrossRef] [PubMed]

(c) 2016 by the author; licensee MDPI, Basel, Switzerland. This article is an open access article distributed under the terms and conditions of the Creative Commons Attribution (CC-BY) license (http://creativecommons.org/licenses/by/4.0/). 